\title{
A Cultura de Segurança no Cuidado Paliativo Oncológico durante a Pandemia de Covid-19
}

https://doi.org/10.32635/2176-9745.RBC.2020v66nTemaAtual.1146

The Culture of Safety in Palliative Oncological Care during the COVID-19 Pandemic
La Cultura de la Seguridad en la Atención Oncológica Paliativa durante la Pandemia Covid-19

Nair Caroline Cavalcanti de Mendonça Bittencourt'; Maria das Graças Gazel de Souza' ${ }^{2}$ Regina Bokehi Nigri'; Vanessa Gomes da Silva ${ }^{4}$

\section{INTRODUÇÃO}

Atualmente, o mundo vive um cenário de pandemia causada pela síndrome respiratória aguda grave do coronavírus 2 (severe acute respiratory syndrome coronavirus 2 - Sars-CoV-2), com impacto na economia, saúde pública e saúde mental de toda a sociedade ${ }^{1}$, cujos profissionais de saúde trabalham para o enfrentamento da doença pelo coronavírus 2019 (coronavirus disease 2019 - Covid-19), arriscando suas vidas e vivenciando situaçóes que geram desgastes físicos e psicológicos².

E, em se tratando de cuidado paliativo oncológico, que tem por objetivo o alívio do sofrimento humano, seus princípios devem ser aplicados tanto de forma individual, diante de uma doença que ameaça à vida, como por exemplo o câncer avançado, quanto diante de uma população que sofre por risco de perda massiva de vidas ${ }^{3}$.

Em tempos de crise, Balsanelli e Cunha ${ }^{4}$ mostram que a sobrecarga relacionada aos longos turnos de trabalho dos profissionais da saúde e o estresse físico e psicológico acabam por deteriorar as bases para a segurança. Ocorre um empobrecimento do corpo funcional, que atua em "modo de crise". Essa sobrecarga leva ao estresse e à fadiga, fatores que comprometem o desempenho e que poderão resultar em erros, falta de comunicação e ajuda mútua, problemas interpessoais, perda do respeito e compreensão.

$\mathrm{Na}$ área da saúde, destaca-se o movimento mundial acerca do tema segurança após a divulgação do estudo "Errar é humano: construindo um sistema de saúde mais seguro" (To err is Human: building a safer health system), em $1999^{5,6}$.

No Brasil, a segurança do paciente entrou na agenda política desde a mobilização da Agência Nacional de
Vigilância Sanitária (Anvisa) do Ministério da Saúde (MS) junto à Organização Mundial da Saúde (OMS) para alcance dos objetivos ${ }^{6}$.

A segurança é o primeiro passo para melhorar a qualidade da assistência prestada; contudo, para tal, é necessária a motivação intrínseca dos profissionais, lembrando que "Errar é humano, mas os erros podem ser evitados"s.

Os sistemas de saúde devem afastar-se da cultura de "culpa e vergonha" que impede o reconhecimento de erro, obstruindo a possibilidade de aprender com ele ${ }^{7}$. Recursos devem ser investidos para a divulgação das experiências, o que pode ser muito lucrativo para pacientes e profissionais, além de resultar em economia para o sistema de saúdes.

De acordo com Nieva e Sorra ${ }^{7}$, o maior desafio para o avanço em direção a um sistema de saúde seguro está na mudança da cultura institucional de culpar o indivíduo pelos erros individualmente, estes não devem ser tratados como falhas pessoais e sim como oportunidades para melhorar o sistema e evitar danos.

\section{DESENVOLVIMENTO}

Visando a aprimorar a coordenação, a cooperação e a solidariedade global para mitigar a propagaçáo do vírus, a OMS declarou que o surto do novo coronavírus constitui uma emergência de saúde pública de importância internacional ${ }^{8} \mathrm{e}$ foi responsável pelo significativo aumento no número de casos que demandam internação hospitalar, gerando preocupaçóes quanto ao colapso do sistema de saúde?.

Profissionais da saúde reportaram medo de contrair a doença, de transmiti-la a seus familiares, do sofrimento

'Enfermeira. Mestranda da Escola de Enfermagem Anna Nery da Universidade Federal do Rio de Janeiro (EEAN/UFRJ). Unidade de Cuidados Paliativos do Hospital do Câncer (HC IV). Instituto Nacional de Câncer José Alencar Gomes da Silva (INCA). Rio de Janeiro (RJ), Brasil. Orcid iD: https://orcid.org/0000-0002-4451-6258

${ }^{2}$ Enfermeira. Doutora pela Faculdade de Enfermagem da Universidade do Estado do Rio de Janeiro (Uerj). Unidade de Cuidados Paliativos do HC IV/INCA. Rio de Janeiro (RJ), Brasil. Orcid iD: https://orcid.org/0000-0002-6247-9331

${ }^{3}$ Médica. Mestranda do Centro de Ciências Biológicas da Saúde da Universidade Federal do Estado do Rio de Janeiro (Unirio). Unidade de Cuidados Paliativos do HC IV/INCA. Rio de Janeiro (RJ), Brasil. Orcid iD: https://orcid.org/0000-0002-2387-243X

${ }^{4}$ Enfermeira. Doutoranda no Programa de Pós-Graduação Stricto Sensu do Instituto Oswaldo Cruz, Ensino de Biociências em Saúde. Unidade de Cuidados Paliativos do HC IV/INCA. Rio de Janeiro (RJ), Brasil. Orcid iD: https://orcid.org/0000-0003-3438-3401

Endereço para correspondência: Nair Caroline Cavalcanti de Mendonça Bittencourt. Rua Visconde de Santa Isabel, 274 - Vila Isabel. Rio de Janeiro (RJ), Brasil. CEP 20560-121. E-mail: ncarolinne@yahoo.com.br 
por estarem afastados de seus lares, estresse, sensação de perda de controle, desvalorizaçáo e preocupaçáo com o tempo de duração da pandemia ${ }^{10}$.

O medo de contaminação por um vírus potencialmente fatal, de origem, natureza e curso pouco conhecidos, acaba afetando o bem-estar psicológico desses profissionais que trabalham no enfrentamento da doença ${ }^{11}$. O estresse e a pressão de lidar com o ofício, acrescidos do risco de adoecer, provocam severos problemas de saúde mental, aumentando o turnouver e a síndrome de Burnout, além de gerar graves problemas como ansiedade e depressáo ${ }^{12}$.

O emprego precoce dos cuidados paliativos oncológicos auxilia os profissionais no atendimento às pessoas portadoras da Covid-19, independentemente de estarem em situação de final de vida, ressaltando que a família precisa ser igualmente acolhida pelos profissionais que, nesse contexto, podem ser convocados a tomar decisóes difíceis. Portanto, cuidar muito bem dos trabalhadores é de suma importância, visto que sua saúde física e mental é condição para se aproximar do outro, fazendo com que se sintam acolhidos e seguros ${ }^{3}$.

Nesse cenário, episódios de erros tendem a aumentar, causando danos à clientela e afetando quanti-qualitativamente as instituiçóes, sendo, para os profissionais, frequentemente relacionados a sentimentos de vergonha, culpa e medo de punições, perdendo a chance de conhecer e tratar adequadamente essas falhas ${ }^{13}$.

Scott et al. ${ }^{14}$ relatam em seu trabalho que profissionais envolvidos em eventos relacionados à segurança do paciente descreveram uma trajetória para recuperação de um estágio pós-trauma elencado em seis etapas: momento de caos e resposta ao acidente; reflexóes intrusivas; recuperação da integridade pessoal; capacidade de suportar as inquisiçóes; ajuda com "os primeiros socorros" emocionais e o movimento: desistir, sobreviver ou prosperar. $\mathrm{Na}$ conclusão deste trabalho, sugeriu-se que as instituiçôes têm o dever de manter treinamentos que promovam suporte emocional para lidar com esses momentos críticos e que tenham a definição clara do que é segunda vítima e a sua prevalência ${ }^{14}$.

Scott et al. ${ }^{14}$ definiram o conceito de segunda vítima como sendo profissionais de saúde envolvidos em evento adverso imprevisto, erro médico e/ou lesão relacionada ao paciente, tornando-se vítimas em decorrência do trauma pelo evento. Com frequência, ocorre sentimento de responsabilidade pelo desfecho do paciente e de falha, subestimando suas habilidades clínicas e base de conhecimento.

Compreender a ocorrência do erro pode estimular notificaçôes e contribuir para mudar o panorama atual de subnotificaçôes. Salienta-se a importância do investimento na cultura de segurança organizacional a partir da disseminação do conceito de segurança do paciente e das discussóes não punitivas sobre o erro humano ${ }^{15}$. Várias publicaçôes relatam experiências de casos de segundas vítimas e traumas nos contextos emocionais, sociais, culturais, espirituais e físicos ${ }^{16}$ (Figura 1).

Também foi descrito um fenômeno em efeito dominó com quatro grupos: paciente e família (primeira vítima), profissionais da área da saúde (segunda vítima), instituição hospitalar (terceira vítima) e pacientes que irão sofrer danos subsequentes (quarta vítima) ${ }^{17}$ (Figura 1).

De acordo com Duarte et al. ${ }^{17}$, as notificaçóes precisam ser estimuladas nas instituiçôes de saúde, facilitando o conhecimento das ocorrências (Figura 2). É fundamental uma gestão que ofereça compreensão e tratamento adequados das ocorrências, sem culpabilizar o profissional, destacando a necessidade de uma análise completa de todo o sistema organizacional ${ }^{13}$. Nos cuidados paliativos oncológicos, essa avaliação se torna imprescindível por se tratar de pacientes mais vulneráveis e suscetíveis a complicaçóes.

A segunda vítima pode reagir de várias formas, sendo mais comuns: culpa, ansiedade, fadiga, frustração; e menos comuns: reviver o evento por desordem traumática pós-estresse, evitar cuidar de pacientes, ter sintomas de ansiedade severa para retornar ao trabalho, depressão e ideias suicidas ${ }^{18}$ (Figura 1).

Denham ${ }^{19}$ propôs que a segunda vítima deveria ter direitos, denominados como "TRUST": tratamento justo; respeito; compreensão e compaixão; apoio; e transparência e oportunidade de melhora (Figura 2).

Considera-se que a notificação sistemática de incidentes e eventos adversos pode promover discussóes interdisciplinares para busca de soluçóes ancoradas em açôes básicas de segurança, visando a evitar recorrência e a identificar lacunas na segurança do paciente ${ }^{20}$ (Figura 2).

Assim, reduzir elementos que dificultam a comunicação efetiva da equipe, além de promover a notificação de incidentes e eventos adversos, constitui meios de aprimoramento e ferramentas gerenciais com vistas à promoção de um cuidado seguro e efetivo ${ }^{16}$ (Figura 2).

A adoção de medidas relacionadas à cultura de segurança organizacional permite que os profissionais se sintam confortáveis para discutir o erro humano ${ }^{15}$.

A premissa básica deve ser a de que os seres humanos são falíveis e os erros esperados, mesmo nas melhores organizaçôes, devem ser vistos como consequência e não como causa, sua origem não está na "perversidade da natureza humana”, mas nos fatores sistemáticos existentes ${ }^{21}$.

\section{CONCLUSÃO}

Durante a pandemia, as instituiçóes de saúde necessitam redobrar sua atenção para medidas que 


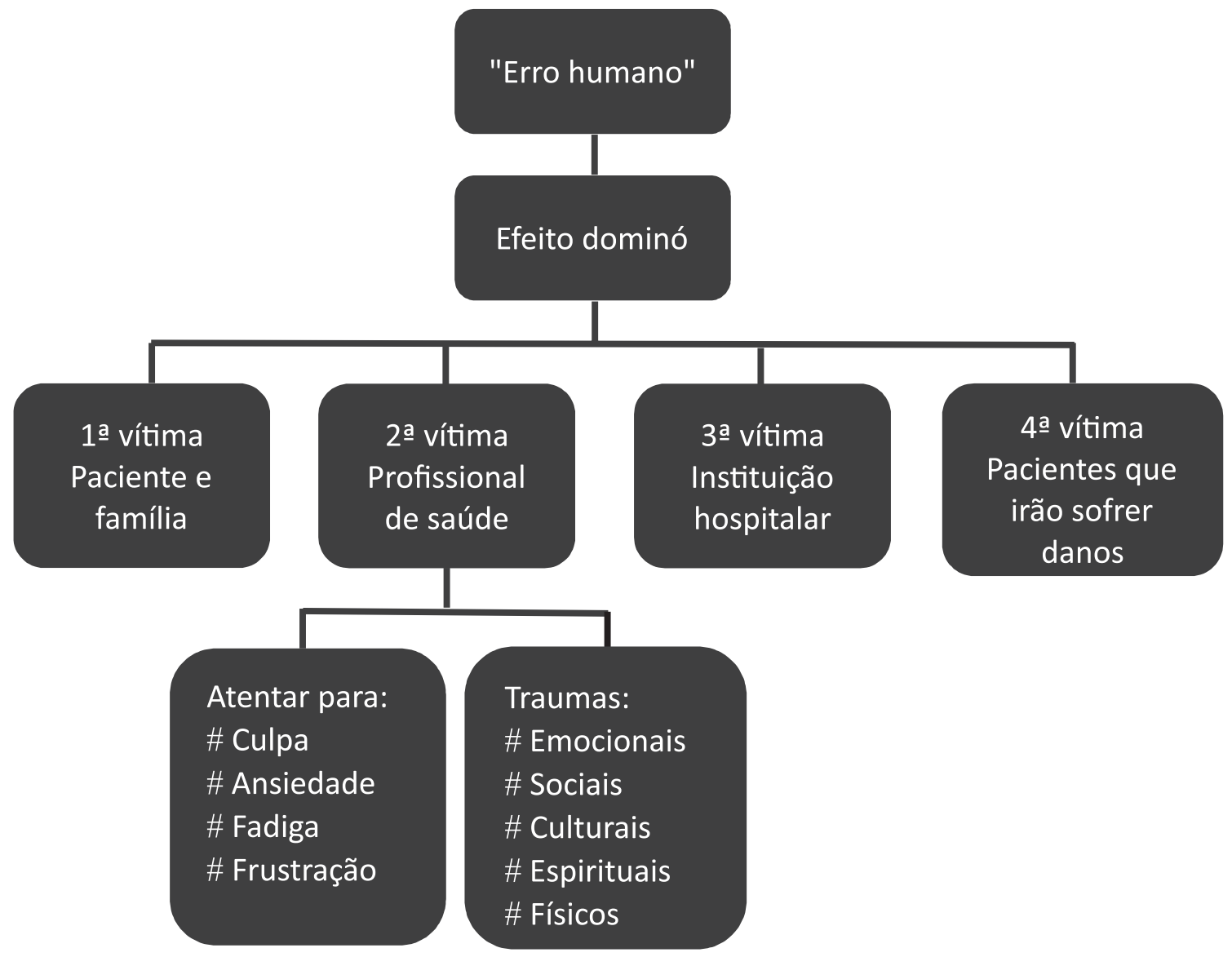

Figura 1. Efeitos do erro humano, 2020

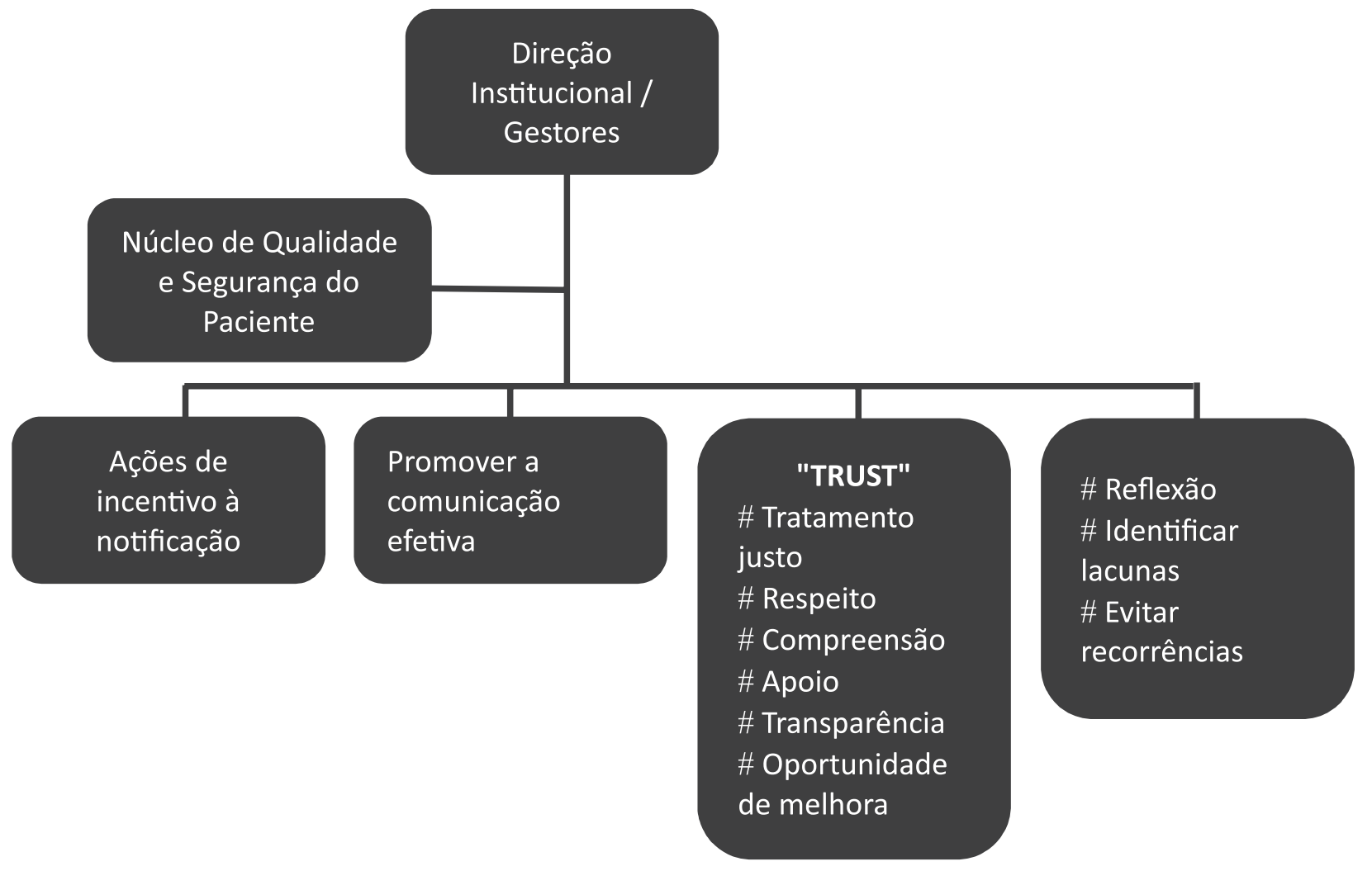

Figura 2. Proposta de abordagem institucional, 2020 
favoreçam o acolhimento dos profissionais e implementar açôes que fortaleçam a cultura de segurança. Nesse cenário, no qual os profissionais de saúde são valiosos, é indispensável a promoção de açôes que protejam a sua saúde.

O Núcleo de Qualidade e Segurança do Paciente, ancorado na reflexão proposta, sugere que a referida orientação contribua para o estímulo de ações gerenciais com vistas a enfatizar o desenvolvimento da cultura de segurança.

\section{CONTRIBUIÇÕES}

Todas as autoras contribuíram na concepção e/ou no planejamento do estudo; na obtenção, na análise e na interpretaçáo dos dados; assim como na redaçáo e revisão crítica; e aprovaram a versão final a ser publicada.

\section{DECLARAÇÃO DE CONFLITO DE INTERESSES}

Nada a declarar.

\section{FONTES DE FINANCIAMENTO}

Não há.

\section{REFERÊNCIAS}

1. Medeiros EAS. Challenges in the fight against the COVID-19 pandemic in university hospitals. Rev Paul Pediatr. 2020;38:e2020086. doi: https://doi. org/10.1590/1984-0462/2020/38/2020086

2. COVID-19: protecting health-care workers [editorial]. Lancet. 2020;395(10228):922. doi: https://doi. org/10.1016/S0140-6736(20)30644-9

3. Maciel MG. Saúde mental e atenção psicossocial na pandemia Covid-19: cuidados paliativos orientaçóes aos profissionais de saúde [Internet]. Rio de Janeiro: Fiocruz; [2020] [acesso 2020 jun 25]. Disponível em: https://www. fiocruzbrasilia.fiocruz.br/wp-content/uploads/2020/04/ Sa\%c3\%bade-e-Mental-e-Aten\%c3\%a7\%c3\%a3oPsicossocial-na-Pandemia-Covid-19-cuidados-paliativosorienta\%c3\%a7\%c3\%b5es-aos-profissionais-desa\%c3\%bade.pdf

4. Balsanelli AP, Cunha ICKO. Nursing leadership in intensive care units and its relationship to the work environment. Rev Latino-Am Enfermagem. 2015;23(1):106-13. doi: https://doi.org/10.1590/01041169.0150 .2531

5. Institute of Medicine (US), Committee on Quality of Health Care in America; Kohn LT, Corrigan JM, Donaldson MS, editors. To Err is Human: Building a Safer Health System [Internet]. Washington, DC: National Academies Press (US); 2000. [cited 2020 Jun
10]. Available from: https://www.ncbi.nlm.nih.gov/ books/NBK225182/pdf/Bookshelf_NBK225182.pdf

6. Agência Nacional de Vigilância Sanitária (BR). Assistência segura: uma reflexão teórica aplicada à prática [Internet]. Brasília, DF: ANVISA; 2017. Capítulo 1, Montserat-Capella D, Cho M, Lima RS. A segurança do paciente e a qualidade em serviços de saúde no contexto da América Latina e Caribe [acesso 2020 jun 24]; p. 13-17. Disponível em: https://www20.anvisa. gov.br/segurancadopaciente/index.php/publicacoes/ item/caderno-1-assistencia-segura-uma-reflexao-teoricaaplicada-a-pratica

7. Nieva VF, Sorra J. Safety culture assessment: a tool for improving patient safety in healthcare organizations. BMJ Qual Saf. 2003;12(Suppl 2):ii17-ii23. doi: https:// doi.org/10.1136/qhc.12.suppl_2.ii17

8. Organização Pan-Americana da Saúde [Internet]. Washington, DC: OPAS; [data desconhecida]. Folha informativa COVID-19 (doença causada pelo novo coronavírus); 2020 [acesso 2020 jun 24]. Disponível em: https://www.paho.org/bra/index.php?option=com_cont ent\&view=article\&id=6101: $\operatorname{covid} 19 \&$ Itemid $=875$

9. Ferguson N, Laydon D, Nedjati Gilani G, et al. Report 9: impact of non-pharmaceutical interventions (NPIs) to reduce COVID-19 mortality and healthcare demand [Internet]. London: Imperial College COVID-19 Response Team; 2020 Mar 16 [cited 2020 jun 18]. Available from: http://doi.org/10.25561/77482

10. Hall RCW, Hall RCW, Chapman MJ. The 1995 Kikwit Ebola outbreak: lessons hospitals and physicians can apply to future viral epidemics. Gen Hosp Psychiatry. 2008;30(5):446-52. doi: https://doi.org/10.1016/j. genhosppsych.2008.05.003

11. Carvalho PMM, Moreira MM, Oliveira MNA, et al. The psychiatric impact of the novel coronavirus outbreak. Psychiatry Res. 2020;286:112902. doi: https://doi. org/10.1016/j.psychres.2020.112902

12. World Health Organization. Mental health and psychosocial considerations during the COVID-19 outbreak [Internet]. Geneva: WHO; 2020 Mar 18 [cited 2020 Jun 18]. Available from: https://www.who. int/docs/default-source/coronaviruse/mental-healthconsiderations.pdf?sfvrsn=6d3578af_2

13. Reason J. Achieving a safe culture: theory and practice. Work Stress. 1998;12(3):293-306. doi: https://doi. org/10.1080/02678379808256868

14. Scott SD, Hirschinger LE, Cox KR, et al. The natural history of recovery for the healthcare provider "second victim" after adverse patient events. Qual Saf Health Care. 2009;18(5):325-30. doi: https://doi.org/10.1136/ qshc. 2009.032870

15. Duarte SCM, Stipp MAC, Cardoso MMVN, et al. Patient safety: understanding human error in intensive nursing care. Rev Esc Enferm USP. 2018;52:e03406. doi: https://doi.org/10.1590/s1980-220x2017042203406 
16. Coughlan B, Powell D, Higgings MF. The second victim: a review. Eur J Obstet Gynecol Reprod Biol. 2017;213:11-16. doi: https://doi.org/10.1016/j. ejogrb.2017.04.002

17. Duarte SCM, Bessa ATT, Büscher A, et al. Error characterization in intensive care nursing. Cogitare Enferm. 2016;21(5 n. esp):01-07. doi: https://doi. org/10.5380/ce.v21i5.45502

18. Romero MP, González RB, CalvoMSR, et al. A segurança do paciente, qualidade do atendimento e ética dos sistemas de saúde. Rev Bioét. 2018;26(3):333-42. doi: https://doi.org/10.1590/1983-80422018263252

19. Denham CR. TRUST: the 5 rights of the second victim. J Patient Saf. 2007;3(2):107-19. doi: https://doi. org/10.1097/01.jps.0000236917.02321.fd

20. Ministério da Saúde (BR). Portaria no 529 , de $1^{\circ}$ de abril de 2013. Institui o Programa Nacional de Segurança do Paciente (PNSP) [Internet]. Diário Oficial da União, Brasília, DF; 2013 abr 2. Seção I:43 [acesso 2020 Jun 22]. Disponível em: http://bvsms.saude.gov.br/bvs/ saudelegis/gm/2013/prt0529_01_04_2013.html

21. Reason J. Human error: models and management. BMJ. 2000;320(7237):768-70. doi: https://doi.org/10.1136/ bmj.320.7237.768 